\title{
Dinámicas sociocognitivas de producción y uso social de conocimiento cientifico sobre salud en dos universidades latinoamericanas
}

\section{Socio-cognitive dynamics of production and social use of scientific knowledge in health in two Latin American universities}

Jorge-Andrés Echeverry-Mejía ${ }^{1}$

Universidad Nacional de Córdoba. Córdoba, Argentina

jorgeandresem@gmail.com

ORCID: https://orcid.org/0000-0001-5502-8316

Citar como: Echeverry-Mejía, J. A. (2021). Dinámicas sociocognitivas de producción y uso social de conocimiento científico sobre salud en dos universidades latinoamericanas. Desde el Sur, 13(2), e0023.

\section{RESUMEN}

El objetivo del trabajo apunta a analizar las dinámicas de producción y uso social de conocimiento científico sobre salud a partir de un abordaje sociocognitivo de las trayectorias de investigadores de dos universidades públicas latinoamericanas: la Universidad de Antioquia (Colombia) y la Universidad Nacional de Córdoba (Argentina). El periodo de análisis está comprendido entre 2016 y 2018. Se adopta un enfoque cualitativo, con observación, revisión documental y entrevistas semiestructuradas como herramientas para la recolección de información. Como base conceptual son tomados los aportes del campo de estudios sobre ciencia, tecnología y sociedad. Se proponen cuatro dimensiones de indagación: la dimensión socioinstitucional, los regímenes de investigación científica y tecnológica, la dimensión cognitiva y la utilidad social de los conocimientos científicos. Los hallazgos muestran una importante heterogeneidad en los abordajes sobre los fenómenos asociados a la salud, los cuales presentan

1 Magíster en Ciencia, Tecnología y Sociedad. Profesor de la Facultad de Ciencias Sociales de la Universidad Nacional de Córdoba (Argentina). Integrante del Programa de Investigación, Conocimiento, Tecnología, Innovación y Sociedad del Centro de Investigaciones y Estudios sobre Cultura y Sociedad, CIECS (CONICET y UNC). 
algunos puntos de contacto en torno a la salud colectiva como tradición latinoamericana.

\section{PALABRAS CLAVE}

Investigadores, ciencias sociales, ciencia y sociedad, ciencia de la ciencia, organización de la investigación, Colombia, Argentina

\section{ABSTRACT}

The aim of this work was to analyze the dynamics of production and social use of scientific knowledge in health from a socio-cognitive approach to the trajectories of researchers from two Latin American public universities: the University of Antioquia (Colombia) and the National University of Córdoba (Argentina). The analysis period ran from 2016 to 2018. A qualitative approach was adopted, employing observation, documentary review and semistructured interviews as tools for information gathering. Contributions from areas of study including science, technology and society were adopted as part of the conceptual basis. Four aspects of inquiry were employed: the socio-institutional dimension, scientific and technological research regimes, the cognitive dimension, and the social utility of scientific knowledge. The findings demonstrate a significant heterogeneity in approaches to the phenomena associated with health, indicating certain points of contact with regard to collective health as a Latin American tradition.

\section{KEYWORDS}

Researchers, social sciences, science and society, science of science, organization of research, Colombia, Argentina

\section{Introducción}

La investigación que dio origen a este artículo (Echeverry-Mejía, 2019) indagó sobre las dinámicas de producción y uso social de conocimientos científicos sobre salud, a partir de la observación y el análisis del trabajo de investigadores de las ciencias sociales y humanidades de dos universidades públicas seleccionadas, una en Argentina y otra en Colombia. El trabajo se concentra en un análisis sociocognitivo que permite aproximarse a la situación actual de la investigación social en salud en dos contextos situados. 
La problematización sobre las determinaciones sociocognitivas que están presentes en los procesos de producción de conocimiento científico sobre salud en las ciencias sociales y humanidades abarca una serie de indagaciones que dan lugar a la investigación en la que se basa este trabajo. Se propone abordar estas cuestiones a partir de los siguientes aspectos: la dimensión socioinstitucional, los regímenes de investigación científica y tecnológica, la dimensión cognitiva y la utilidad social de los conocimientos científicos.

Por un lado, la dimensión socioinstitucional apunta al reconocimiento de las formas específicas de organización de la investigación científica en las dos instituciones seleccionadas, y a partir de allí considerar las estructuras predominantes, los tipos de actores implicados y las configuraciones de sus políticas y estructuras. Por otro lado, los regímenes de investigación científica y tecnológica permiten dar cuenta de las características de las diferentes tradiciones disciplinarias a partir de las identificaciones alrededor de los objetos de estudio, las formas de división del trabajo, los mecanismos de construcción y reproducción de conocimientos y relaciones. Además, en los procesos de investigación está presente una dimensión cognitiva que se refiere a las preocupaciones conceptuales de los actores, el tipo de conocimiento que se quiere producir y el contenido de dicho conocimiento, en este caso asociado a los abordajes de la salud realizados dentro del conjunto heterogéneo que representan las ciencias sociales y humanidades. Finalmente, se alcanza a abordar la dimensión de utilidad social de los conocimientos, con la mirada puesta en el interior de los procesos de producción científica y no solo en los resultados efectivos o visibles.

Las anteriores dimensiones e indagaciones para el análisis fueron sostenidas bajo el supuesto de partida de que los procesos de producción de conocimiento tienen determinaciones sociocognitivas (Knorr Cetina, 2005; Kreimer, 2003; Kreimer et al., 2004; Shinn, 2008; Vaccarezza y Zabala, 2002). Por lo tanto, dichos procesos no son autónomos o separados de la sociedad. Esto significa que el conocimiento logrado mediante la investigación sobre salud, realizada por los investigadores participantes en el estudio, no solo es producto de los aportes y validaciones realizadas por la comunidad disciplinaria internacional o por los cánones de cada campo académico, sino también por las interacciones sociales y por el reconocimiento de los investigadores como sujetos que forman parte de un entramado sociocultural donde hay un flujo de múltiple vía que da lugar a dichos conocimientos $y$ a determinadas representaciones de las problemáticas asociadas, en este caso, a la salud. 
El proyecto que dio origen a la investigación empezó asignando mayor énfasis a la promoción de la salud como concepto y como práctica. El propósito era lograr una aproximación desde el aporte del campo de estudios sobre ciencia, tecnología y sociedad (CTS) a dicha construcción, que actualmente se discute en los ámbitos académico, social, gubernamental y hasta empresarial. Sin embargo, al profundizar en el estado de la cuestión, en la revisión de documentación para la construcción del marco conceptual y en el diseño metodológico, surgieron diferentes concepciones que mostraban no solo una versión sobre el significado de la salud, sino varias.

Al encontrar que la promoción de la salud opera en mayor medida en escenarios políticos y normativos - y que su perfil está más asociado a las acciones y estrategias-, fue realizado un ajuste al objetivo general, que centró la atención en el análisis de las dinámicas de producción y uso social de conocimientos científicos sobre salud. Esto reorientó la atención hacia el trabajo de los investigadores y las condiciones en las que desarroIlan sus actividades en la Universidad de Antioquia (UdeA) y en la Universidad Nacional de Córdoba (UNC).

De esta manera, se buscó partir de las nociones básicas de salud de los investigadores antes de considerar aspectos referidos a su promoción, puesto que se empieza desde el reconocimiento de que las definiciones condicionan lo que posteriormente se concreta en la intervención o acción. Es decir, la forma en que se está entendiendo la salud influye en las decisiones sobre acciones de promoción, las cuales, actualmente, según revelan los propios investigadores y organismos sanitarios, están orientadas hacia el control de factores de riesgo y el fomento de «hábitos saludables», en línea con las visiones institucionales propuestas e impulsadas por agendas internacionales y gobiernos. En este sentido, se parte del postulado de que los esquemas científico-sociales modelan el mundo y orientan las prácticas (Lamo de Espinosa, 2005).

Para lograr el análisis propuesto se procedió con la caracterización de las áreas de la salud de la UdeA y la UNC, a partir de la observación de sus estructuras institucionales y la revisión de políticas universitarias y otras fuentes especializadas en la organización de las actividades de investigación. Posteriormente, se recurrió a la identificación y el análisis de la situación de los investigadores sociales dedicados a la producción de conocimiento sobre salud, considerando particularmente sus agendas de investigación y los abordajes que realizan sobre la salud, la enfermedad y la promoción de la salud. Finalmente, fue realizado el análisis de las posturas de los investigadores sobre las condiciones de producción científica, complementadas con la información obtenida de los momentos anteriores. 
La investigación aporta, por un lado, un abordaje integrador a partir del enfoque ofrecido por el campo académico de estudios de CTS. Esto implica un ejercicio de análisis interdisciplinar, puesto que toma herramientas de varias tradiciones disciplinarias para lograr una aproximación a los procesos sociocognitivos en torno a la salud como objeto de estudio y como ámbito de prácticas. Por otro lado, el trabajo constituye un aporte para los investigadores participantes, sus colegas, las comunidades disciplinarias que estudian la salud y las instituciones seleccionadas, dado que ofrece un panorama de la investigación social de la salud y propone un ejercicio de reflexividad tanto desde la implementación como desde los resultados. Finalmente, para el campo CTS también constituye un aporte, ya que presenta una base analítica acerca de las determinaciones sociocognitivas de los procesos de producción de conocimiento científico sobre un tema u objeto de estudio complejo, como lo es la salud. Esto considerando además a las ciencias sociales y humanidades como base de análisis y no a las ciencias exactas y naturales, que han sido las que mayor atención han recibido en este tipo de abordajes. Como afirma Quiroz Ávila (2018), el valor de las ciencias sociales y humanidades es central, particularmente en nuestros países, por lo que se requiere mayores esfuerzos por comprender los aportes que representan para la comunidad académica y la sociedad en su conjunto.

Un abordaje CTS implica una perspectiva integradora, sociológica, histórica, política, ética o económica, y también articulada, tomando elementos de utilidad de diferentes propuestas conceptuales para lograr un resultado, o al menos una aproximación, interdisciplinar. En América Latina en particular, estos estudios se desarrollan desde la década de 1960 y con mayor consolidación desde 1980, con la conformación de grupos de investigación, encuentros especializados y posgrados específicos (Kreimer y Thomas, 2004). Entre los ejes temáticos que abarca este campo se encuentran: dinámica de campos científicos, migraciones científicas, relaciones universidad-empresa, ciencia e inclusión social, estudios de laboratorio, relaciones centro-periferia, producción y uso de conocimientos, entre otros (Kreimer et al., 2014).

En línea con los análisis de producción y uso de conocimientos, se toma como referencia principal la conceptualización de los regímenes de investigación científica y tecnológica, desarrollada por Shinn (2008), que aborda la dinámica de los campos a partir del conjunto de relaciones sociocognitivas que los componen y de la articulación de diversos actores alrededor de una disciplina en común. La teoría transversalista de la producción y difusión de la ciencia y la tecnología es explicada por Shinn a partir de cuatro regímenes: régimen disciplinario, régimen utilitario, régimen 
transitorio y régimen transversal. «Cada régimen tiene su base histórica, posee su propia división de trabajo, sus propios modos de producción cognitiva y de artefactos y tiene audiencias específicas» (Shinn, 2008, p. 11).

A grandes rasgos, el régimen disciplinario se refiere a las prácticas consolidadas de la investigación científica desde el siglo XIX, que aún continúa vigente y en expansión —más allá de la retórica de un «nuevo» modo de producción de conocimientos (Gibbons et al., 1994) - y se ve reflejado en el modelo alemán humboldtiano con una universidad dividida en facultades con el propósito de formación e investigación en las ciencias, lo que se sostiene en el carácter dual de la docencia y la investigación (Burke, 2002, 2012). El régimen utilitario está representado por las escuelas de ingeniería; mientras que el anterior se centra en la comprensión, este se inclina por la utilidad, por los artefactos y una consideración económica dominante. El régimen transitorio representa mayores interacciones entre el ámbito académico y el productivo, pero no es una vinculación sostenida y sigue siendo el régimen disciplinario el referente primario. Finalmente, el régimen transversal es una conjunción de varios sectores socioproductivos, dado que se desarrollan travesías entre las fronteras disciplinares y se mantiene una vinculación fluida e interdependiente; en este régimen se destaca la investigación tecnológica y su capacidad de promover la convergencia entre grupos heterogéneos, además de representar un modo de producción científica distinto (Shinn, 2008).

Ante la diversidad que representa la producción de conocimientos científicos, Shinn propone los diferentes regímenes e incluso prefiere hablar de ciencias y no solo de ciencia en singular. Sin embargo, su propuesta está muy enfocada en ciencias naturales y su relación con la investigación tecnológica. Aun teniendo en cuenta esta cuestión, es tomado para este trabajo, dado el peso que representa el régimen disciplinar en las dos universidades seleccionadas coincidiendo con las descripciones que presenta el autor.

Además de considerar los regímenes de producción, también es importante la problematización sobre la construcción de la utilidad social del conocimiento. En este sentido, la propuesta que desarrolla Kreimer (2003) se relaciona con los aportes de Shinn y se concreta en múltiples formas que a continuación se detallan y que se refieren a la visión de utilidad desde el punto de vista de los investigadores mismos sobre su trabajo:

- Desarrollar sus investigaciones sin tomar en consideración ningún tipo de usuario o de actor otro que los propios colegas de un campo disciplinario. Esta práctica es característica, en principio, de lo que Shinn denomina «régimen disciplinario», entendido de un modo tradicional. 
- Incorporar, en el curso de la construcción de una «agenda de investigación», la representación de un usuario «ideal». Este actor, idealmente construido, puede ignorar los productos del conocimiento, localizados en una interacción imaginaria. Este tipo de práctica, también propia de regímenes disciplinarios, puede estar influida por políticas de estímulo a la innovación, como por motivaciones propias de los grupos de investigación, en la búsqueda de legitimación de sus propios conocimientos. Este caso es uno de los que caracteriza la emergencia del fenómeno CANA ${ }^{2}$.

- Una variante específica del caso precedente se produce cuando el productor de conocimientos, a pesar de la construcción ideal de un usuario ideal, logra interactuar efectivamente con aquel. Puede entonces comenzar un mecanismo de resignificación del producto, tanto como del proceso mismo de investigación y puede (o no) dar lugar a una utilidad efectiva. Este caso es propio de regímenes de transición, aunque, luego de las relaciones entabladas por los diferentes actores, puede igualmente dar lugar a fenómenos de CANA.

- Finalmente, otros actores significativos pueden estar presentes de un modo real y efectivo, $a b$ initio, en los procesos de producción de conocimientos. Estos actores participan en los aspectos cognitivos de la producción científica a través de un proceso muy complejo. Normalmente, estos procesos - muy poco frecuentes en los países periféricos - dan lugar a una utilización efectiva de los conocimientos producidos. Puede hacer «entrar» o «salir» a otros actores, en un proceso dinámico. Estos procesos son, por regla general, específicos de los regímenes «transversales» (Kreimer, 2003, s. p.).

Si bien las ciencias sociales y humanidades, dadas sus diferencias con las ciencias exactas y naturales en la construcción de su objeto de investigación, podrían llegar a un régimen transversal, esto no fue hallado en las unidades de análisis. Prevaleció el régimen disciplinario dentro de las instituciones académicas, o con algunas experiencias propias del régimen de transición cuando los investigadores lograron algún un tipo de interacción con otros actores, pero no de forma sostenida en el tiempo, sino más bien circunstancial. Una de las razones que podría estar impulsando esta situación es que, por más que las ciencias sociales y humanidades realicen problematizaciones locales, su configuración disciplinar sigue respondiendo a una lógica sociocognitiva en la que la comunidad disciplinaria internacional logra tener un peso importante sobre la construcción de las agendas de investigación, los criterios cognitivos y

2 Se trata de Conocimiento Aplicable No Aplicado, fenómeno propio y extendido en la mayor parte de los países periféricos. Para un análisis en profundidad de este fenómeno, véase Kreimer y Thomas $(2002,2003)$. 
las metodologías. Esto se ve reflejado, por ejemplo, en la reproducción de líneas de investigación y autores provenientes de países centrales, que se sostienen como referencia principal. Por otra parte, entran también en juego los sistemas de «recompensas y sanciones» traducidos en los procesos de evaluación y promoción laboral, lo que repercute en una apuesta, por parte de los investigadores, por un terreno seguro que les garantice su continuidad y avance disciplinar (Vasen, 2018).

El uso o utilidad social del conocimiento está atravesado, entonces, al decir de Zabala (2010, 2014), por los condicionamientos que impone la organización social en cada momento, tanto en el orden de la configuración científica como en el plano político. Esto aporta, además, al paralelo que se genera entre la forma en que un fenómeno se constituye, ya sea como un problema científico o un problema social, o como ambas cosas. La producción de conocimiento científico puede llegar a operar en la definición o imposición de temas en las agendas política y pública, así como dichas agendas y problemas sociales pueden llegar a operar en la configuración de las agendas de investigación.

También es importante considerar que, tradicionalmente, la referencia hacia el uso y la utilidad de la investigación ha estado muy asociada a las ciencias exactas y naturales. En este sentido, es importante el aporte de Vaccarezza (2009), que aborda el tema desde las ciencias sociales y humanidades, dando cuenta de una mayor heterogeneidad y variabilidad y proponiendo cinco modelos de intercambio que apuntan a lo que sucede, particularmente, con los resultados de la investigación en las áreas sociales:

a) Modelo de intercambio 1: resultados por acceso

b) Modelo de intercambio 2: facilidades de profesionalización y mantenimiento del grupo de trabajo

c) Modelo de intercambio 3: venta de servicios

d) Modelo de intercambio 4: investigación por prestigio

e) Modelo de intercambio 5: intercambio de reflexividad

Considerando este análisis, el autor propone además una clasificación de la «orientación pragmática del investigador», dando así lugar a tres posibilidades: los investigadores con orientación fundamentalmente académica, aquellos que están orientados hacia la consultoría comercial y finalmente los involucrados en procesos de transformación social (investigación con intervención).

Todo este abordaje no debe dejar de tener en cuenta la relación centro-periferia en la investigación científica, puesto que, si bien hay ciertas líneas de regularidad por la aparente alineación internacional de criterios 
científicos, las formas de producción y circulación de conocimientos vienen siendo muy condicionadas por las dinámicas y capacidades de los países centrales, lo que genera efectos sobre la configuración de las agendas de investigación de los países periféricos. Incluso esto produce una suerte de injerencia y concentración, así como también lo que Vessuri, Guédon y Cetto (2014) denominan como el efecto de ayuda externa en sentido inverso, en el que los países y grupos periféricos terminan adoptando una posición funcional, respondiendo y aportando a los avances de investigación de interés para las agendas centrales y descuidando de esa forma el abordaje de problemáticas propias de países del sur global.

\section{Aspectos metodológicos}

Los estudios CTS, además de brindar herramientas conceptuales para analizar las diferentes lógicas de configuración de la investigación científica y sus actores, también ofrecen aportes metodológicos al indagar, de manera interdisciplinaria, sobre las dinámicas de producción, circulación, validación y uso de los conocimientos (Kreimer y Thomas, 2003). En definitiva, un análisis como el presentado en esta oportunidad, intenta dar cuenta de un ejercicio, al decir de Bourdieu (2003), de ciencia de la ciencia y reflexividad, puesto que ingresa al mundo científico buscando comprenderlo desde la voz de sus propios actores, desde la revisión de sus lógicas, desde la literatura especializada y desde los contextos sociales y cognitivos. Además, busca generar un aporte a la reflexividad, no solo desde el procesamiento de los resultados y los hallazgos, sino también desde el proceso mismo de investigación. En este caso, al momento de abordar a los investigadores se les preguntaba sobre su trayectoria profesional, las motivaciones que los conducían a investigar, además de los detalles propios de su forma de abordar la salud como objeto de estudio.

El presente trabajo vincula dos problemáticas. Por un lado, la poca reflexión y análisis sobre los procesos y resultados del trabajo intelectual de investigadores sociales, particularmente para este caso, los dedicados al abordaje de la salud; $y$, por otro lado, la concepción que tienen estos investigadores sobre su objeto de estudio y lo que representarían sus aportes para una comprensión diferente de la salud y su promoción.

Para realizar el análisis se partió de un enfoque cualitativo, puesto que este aporta al logro de los objetivos planteados en la investigación. De allí que la caracterización institucional de las dos universidades (particularmente las áreas asociadas a la salud), la identificación de los actores que trabajan sobre salud (los investigadores) y sus interpretaciones sobre la salud, la enfermedad y la promoción de la salud fueran posibles, dado que este tipo de enfoque facilita la comprensión de razones, lógicas, 
racionalidades, visiones, modos de ser y de comportarse de múltiples actores sociales en su diversidad y heterogeneidad social (Galeano Marín, 2004).

La estrategia metodológica adoptada para este trabajo se basa en el estudio de casos múltiples (Hernández Sampieri et al., 2014), y corresponde, en este caso, con dos instituciones: la UdeA y la UNC. Cada caso se presenta como un agrupamiento de investigadores sociales en salud. Esto no significa que sean integrantes de un mismo equipo, ni siquiera de una misma facultad o dependencia académica, sino que coinciden en un abordaje de la salud desde marcos conceptuales y metodológicos provenientes de las ciencias sociales y humanidades. Son tomados entonces como dos casos complejos donde hay una unidad de análisis representada por las dos universidades públicas elegidas $y$, a su vez, subunidades equivalentes a los investigadores con afiliaciones a diferentes dependencias y agendas de trabajo, lo que comprende aspectos de la trayectoria personal y profesional y el tipo de vínculo con la institución.

Las técnicas aplicadas para la obtención de información son: observación de campo, relacionada con los contextos socioinstitucionales de las dos universidades (políticas y estructura institucional, condiciones externas); revisión documental de fuentes institucionales y bibliografía especializada basada en registros y normativas referidas al funcionamiento de la investigación tanto en las dos instituciones como en los dos países; además de bibliografía sobre salud que iba surgiendo de las menciones de los entrevistados y de la consulta de bases de datos especializadas como SciELO y Redalyc. También fueron realizadas entrevistas semiestructuradas que contaron con una guía de preguntas para facilitar cierta regularidad en el abordaje de todos los informantes, pero dando lugar a mayor flexibilidad para lograr una aproximación más conversacional y fluida a los aspectos y temas en consideración. El tipo de análisis para la información obtenida fue de tipo documental, considerando datos internos (institucionales, normativos) y externos (a partir de las relaciones entre los documentos y su medio cultural); además del análisis interpretacional sobre las declaraciones de los investigadores y las condiciones institucionales y sociales de producción y uso de conocimiento científico.

La investigación fue realizada entre 2016 y 2018. Dicho margen temporal fue elegido partiendo de la indagación por la situación más reciente, de manera que fuera posible brindar un análisis de situación actual.

Hay varias razones para decidir la focalización sobre investigadores sociales y no sobre todos aquellos que forman parte de las denominadas áreas de la salud en cada universidad. En primer lugar, por el alcance de la investigación. Se buscaba dar cuenta de una aproximación descriptiva y explicativa que mostrara algunas características de la investigación en 
salud desde la perspectiva de las ciencias sociales y humanidades, presentes en las dos instituciones en diferentes formas de organización estructural. Además, se suma la decisión de dar cuenta de un abordaje poco trabajado y por tanto poco visible, frente a un estudio tradicional de la salud asociado a las Facultades de Ciencias Médicas y a profesionales biomédicos, que han tenido mayor reconocimiento, visibilidad y legitimidad en el ámbito de la salud. Su discurso es el que más se ha considerado y naturalizado, por lo cual la problematización que se propone desde este trabajo da lugar a que sean los investigadores de las ciencias sociales y humanidades los que ofrezcan sus perspectivas y dimensiones.

La selección de las dos universidades se realizó por conveniencia, frente a la factibilidad de acceso a la información y a los investigadores. La cercanía a estas instituciones permitió desarrollar la observación de campo, la revisión documental y la realización de entrevistas.

Cabe resaltar las dificultades representadas por la desactualización de los sitios web y la falta de mecanismos estandarizados y funcionales para la obtención de datos sobre perfiles de investigadores y grupos, líneas de investigación y actividades asociadas a la producción y uso de conocimientos científico-tecnológicos.

En el caso de la UNC se dificultó más la recolección de información, dado que la unidad básica de organización de sus actividades de investigación no se basa en grupos sino en individuos. Incluso, sobre ellos, es difícil acceder a la información correspondiente puesto que la visualización pública que ofrece el Sistema Integral de Gestión y Evaluación (Sigeva) no permite búsquedas avanzadas, contiene información incompleta y no facilita el contacto. Otra opción presentada por la Secretaría de Ciencia y Tecnología de la UNC (SeCyT-UNC) es la consulta por proyectos de investigación; sin embargo, también aparece incompleta, sin palabras clave, resumen o acceso al proyecto; además, no es posible encontrar interconexión entre el director, los integrantes de los proyectos y sus perfiles en el Sigeva. Tampoco aparecen detalles sobre tipo, estado o fechas asociadas a los proyectos. Además, el catálogo de publicaciones con subsidio de la SeCyT suele aparecer fuera de servicio. Por otra parte, el CVar, que es un mecanismo nacional centralizado desde el Portal de Información de Ciencia y Tecnología Argentino, presenta fallas constantes, lo que impide consultar el curriculum vitae de los investigadores o algún listado de temas o líneas de investigación en las que trabajan.

En la UdeA el panorama informativo no es mejor. Si bien la organización de la investigación toma como unidad básica a los grupos y esto permite identificar cierta trayectoria sostenida en el tiempo — de integrantes y producción-, es común que los datos estén desactualizados; además, 
la plataforma informática administrada por Colciencias, que brinda información sobre los grupos de investigación (denominado GrupLAC) y el currículum académico (denominado CVLAC), suele estar fuera de servicio, no facilita búsquedas avanzadas y no cuenta con alguna forma de contacto con el investigador.

Si bien estas limitaciones generaron retrasos en la identificación de los investigadores y la realización de las entrevistas, fueron logrados 29 encuentros distribuidos en partes relativamente iguales para cada universidad. La determinación de la cantidad no fue predefinida y el límite fue declarado por saturación de categorías.

Para esta investigación el rol de las entrevistas fue central, puesto que condujo a la ampliación de fuentes y bibliografía especializada, a profundizar la observación de campo relacionada con el trabajo de los investigadores y el contexto socioinstitucional en el que se desarrolla, y además ofrecía elementos que usualmente pasan desapercibidos en los tradicionales estudios cuantitativos más centrados en la bibliometría o en indagaciones realizadas a partir de documentos oficiales. Como indica Vasen (2018), el enfoque cualitativo y la realización de entrevistas o etnografías para análisis de la producción intelectual permite comprender las señales que reciben (y que generan) los investigadores desde y hacia el sistema académico-científico y los circuitos en los que participan. Por esto, la presente investigación no se centra en identificar o analizar la cantidad y frecuencia de las publicaciones sobre salud, sino en conocer las visiones de los investigadores desde la reflexión sobre sus prácticas.

La guía de entrevistas fue diseñada con 25 preguntas que buscaron dar cuenta de la trayectoria personal y profesional de los investigadores, en línea con el análisis sobre las dinámicas de producción y uso social de conocimiento científico. A continuación se presentan algunos resultados.

\section{Resultados}

Los contextos socioinstitucionales de las dos universidades elegidas para este estudio son diferentes y al mismo tiempo coinciden en relación con algunas prácticas académico-científicas. Estas instituciones están amparadas en la lógica de la autonomía frente a su funcionamiento interno. Sin embargo, también reciben una fuerte influencia de sus entornos locales, nacionales e internacionales y de las estructuras regulatorias que definen las políticas educativas y científicas.

Particularmente en materia de investigación científica y tecnológica, las posibilidades de desarrollo de capacidades y actividades depende de las políticas y estructuras institucionales dedicadas al tema. Uno de los aspectos clave está representado en el financiamiento, que se traduce tanto 
en la disponibilidad de personal contratado, como de las opciones efectivas de realización de investigaciones.

Colombia es uno de los países que menos invierte en Investigación y Desarrollo (I+D) en la región: $0,27 \%$, por debajo de Costa Rica $(0,46 \%)$ Ecuador $(0,44 \%)$, Uruguay $(0,41 \%)$, Chile $(0,36 \%)$, Cuba $(0,34 \%)$ y Venezuela (0,32\%) (RICYT y OCTS-OEI, 2018).

La organización de la estructura científico-tecnológica colombiana se basa principalmente en las capacidades del sistema universitario, que cuenta con 31 universidades públicas y 53 universidades privadas, sin mencionar otras modalidades de instituciones de educación superior (IES), dado que, si se totalizaran - agregando instituciones universitarias, tecnológicas y técnicas-, la equivalencia sería de 62 IES públicas, 211 IES privadas y 19 de régimen especial. Adicionalmente, hay que tener en cuenta que las instituciones privadas colombianas, particularmente las universidades, tienen importantes fortalezas en investigación y compiten por recursos en la materia a través de contrataciones o participación en convocatorias públicas.

Específicamente, en el caso de la UdeA se puede encontrar, a grandes rasgos, que, si bien esta casa de estudios fundada en 1803 es de orden departamental, está sujeta a la jurisdicción nacional a través del Ministerio de Educación Nacional y al Sistema Nacional de Ciencia, Tecnología e Innovación. Su población es cercana a los 37000 estudiantes de grado, 3200 estudiantes de posgrado, 1690 «profesores vinculados» (UdeA, 2016), y unos 5600 «profesores de cátedra» (personal con mayor inestabilidad, sin vínculo fijo y mayor vulnerabilidad laboral).

La UdeA cuenta con 15 unidades administrativas que componen el Comité Rectoral, entre las que se encuentra la Vicerrectoría de Investigación, que lidera el Comité para el Desarrollo de la Investigación (CODI) y el Sistema de Investigación de la universidad. Este sistema, a su vez, está compuesto por 25 centros de investigación radicados en las unidades académicas o existentes de forma independiente. Además, la gestión de la investigación presenta una división en cuatro grandes áreas (adaptando el modelo de la OCDE): ciencias sociales, humanidades y artes (cuenta con 11 centros de investigación); ciencias médicas y de la salud (10 centros); ingeniería y tecnología ( 2 centros); ciencias exactas y naturales ( 1 centro); además de la Sede de Investigación Universitaria (división especial para grupos de investigación consolidados).

La investigación en la UdeA se rige por el Acuerdo Superior (AS) 204/2001 y el Acuerdo Superior 386/2011 (que modifica algunos puntos del primero). El AS 204/2001 sustituye al AS 153 de 1990, que creó el Sistema 
Universitario de Investigación (SUI), y asimismo reglamenta la actividad de investigación en la universidad, considerando el Estatuto General vigente desde 1994 y forma parte de los planes de desarrollo decenales implementados formalmente desde 1995. Por otra parte, desde 1993 la UdeA integra el Sistema Nacional de Ciencia y Tecnología liderado por Colciencias (hoy MinCiencias), adoptando así las políticas que se definan desde su órbita, lo que se refleja, por ejemplo, en la consideración del Grupo de Investigación Científica y Tecnológica como unidad básica de generación de conocimiento científico y de desarrollo tecnológico, diferente de la forma como se estructura en otros países respecto del investigador individual como unidad central; lo que genera que en el caso de Colombia se hayan hecho procesos de medición, evaluación y clasificación, hasta fechas recientes, de los grupos y no de los investigadores de forma individual.

En el caso del contexto argentino, es posible encontrar que el país ha sido uno de los líderes en América Latina en cuanto a su estructura científico-tecnológica. Si bien en años recientes se redujo el presupuesto en el sector, su presencia venía siendo destacada. La inversión en investigación y desarrollo en 2012 era de un $0,65 \%$ de su PIB, con una proyección hacia 1,01\% en 2020. Sin embargo, cifras de 2016 reflejan una disminución al 0,53\% (OCTS-OEl, 2018). El sector CTI se complementa con su importante sector universitario público, puesto que el $44 \%$ de personas que se dedican a la investigación se desempeñan en las universidades públicas (MinCyT Argentina, 2017, p. 30). Las universidades nacionales públicas y gratuitas suman un total de 55 , distribuidas en todo el territorio nacional, además de existir la Universidad Tecnológica Nacional (UTN, también pública), con 29 sedes nacionales, especializada en ingenierías y tecnologías.

A las universidades nacionales se agrega la presencia del Consejo $\mathrm{Na}-$ cional de Investigaciones Científicas y Técnicas (Conicet) y otras 17 instituciones que también participan en el Sistema Nacional de Ciencia, Tecnología e Innovación.

La ley nacional que da lugar a la reunión de estas instituciones es la 25467 de 2001, conocida como la Ley de Ciencia, Tecnología e Innovación, que formaliza la estructura del Sistema Nacional de Ciencia, Tecnología e Innovación (SNCTI), define las responsabilidades del Estado Nacional y los aspectos referidos a la planificación, el financiamiento y la evaluación de las actividades de investigación y desarrollo.

En Argentina, a diferencia del resto de países de la región, existe la Carrera del Investigador Científico (CIC), administrada, en conjunto con las becas doctorales, por el Conicet. Esto significa la existencia de personal con dedicación exclusiva a labores de investigación, distribuido en cuatro grandes áreas del conocimiento: ciencias agrarias, ingeniería y de materiales; 
ciencias biológicas y de la salud; ciencias exactas y naturales; ciencias sociales y humanidades. Actualmente se desempeñan en Conicet más de 10 000 investigadores y más de 11000 becarios doctorales distribuidos en las 23 provincias argentinas. Además, el Conicet tiene convenios con las universidades nacionales y otros organismos para la gestión conjunta de unidades divisionales, es decir, institutos, laboratorios o centros de investigación y desarrollo de doble dependencia. Es así como cuenta, particularmente en conjunto con la UNC, con 22 unidades ejecutoras de doble dependencia.

Entrando en las particularidades de la UNC, cabe decir que se trata de la institución universitaria más antigua del país y una de las más longevas de América. Con la instalación de estudios superiores desde 1613, es una de las pioneras en la formación superior, y aún en la actualidad tiene un gran reconocimiento académico y social en Córdoba, en el país y en el mundo, debido no solo a su trayectoria institucional, sino también por haber sido el epicentro de la reforma universitaria de 1918, un movimiento democratizador que transformó la forma de entender la educación superior frente a los desafíos sociales, políticos, culturales y económicos de principios del siglo XX (Isoglio y Echeverry-Mejía, 2018). Las repercusiones de la reforma aún se sienten con la defensa de la autonomía universitaria, el cogobierno, los concursos públicos, la vinculación con la sociedad, entre otras demandas por la educación como un derecho emancipador y transformador.

La UNC es un ente autónomo cuya población es estimada de la siguiente manera: unos 117225 estudiantes de grado, 9602 estudiantes de posgrado, 5360 estudiantes de pregrado y 3761 estudiantes de nivel medio. Esto suma un total de 135948 estudiantes en todos los programas universitarios. Además, la UNC cuenta con más de 9000 profesores en varias categorías, de los cuales se tiene registro de alrededor de 3000 que realizan actividades de investigación. La condición de acceso irrestricto, masivo y gratuito representa un importante énfasis en la docencia, considerando además que las universidades argentinas y latinoamericanas en general están emplazadas en contextos marcados por la desigualdad y la necesidad de formación técnica y profesional, a lo que cabe agregar la tradición imperante de universidades de estilo napoleónico, es decir, profesionistas.

La investigación en la UNC está contemplada en su estatuto y la define como una de las misiones básicas, para lo cual se propone la realización de investigaciones científicas, el desarrollo de capacidades tecnológicas y la formación, el mantenimiento y el mejoramiento de los recursos humanos y físicos para la investigación. Además, desde la Ordenanza 15/1988 del 
Honorable Consejo Superior (HCS) se dispuso la creación de la Secretaría de Ciencia y Tecnología (SeCyT-UNC) y la definición del reglamento orgánico que define la estructura y las funciones institucionales en la materia.

\section{Investigadores sociales de la salud}

Para comprender las dinámicas de producción y uso de conocimiento científico resulta importante conocer la manera en que los investigadores inician su trayectoria, particularmente en relación con sus indagaciones sobre la salud. En este sentido, los informantes relataron las circunstancias por las cuales llegaron al campo de la salud, entre las que se encuentran: oportunidades laborales, posiciones políticas sobre el derecho y la defensa de la salud, los antecedentes familiares, el incentivo de docentes, entre otras.

La aproximación para caracterizar los perfiles de los investigadores entrevistados aportó en la definición de las categorías de análisis: ¿qué investigan? (tema dentro del gran área de salud), ¿cómo investigan? (enfoques metodológicos), ¿por qué investigan? (motivaciones), ¿para qué y para quiénes investigan? (usos de las investigaciones y sus resultados).

A continuación, se presenta una caracterización de los informantes a partir de los resultados obtenidos en las entrevistas: los entrevistados fueron un total de 29, 9 hombres y 20 mujeres. La cantidad de investigadores entrevistados corresponden, en partes relativamente iguales: 14 pertenecen a la UdeA y 15 pertenecen a la UNC.

Las disciplinas en las que trabajan los investigadores son muy variadas; aparecen: Trabajo Social, Psicología, Comunicación, Sociología, Historia, Economía, Ciencia Política, Administración de Salud, Antropología, Demografía, Filosofía y Educación. En varios de los casos no se reflejan las formaciones de base, sino los campos académicos en los que se respaldan para realizar sus análisis, incluso combinando aportes para lograr un abordaje más interdisciplinario de sus problemas o temas de investigación.

Definiendo dos grandes bloques de la población objeto de estudio - uno representado por las ciencias sociales, humanidades y artes (CSHA), y otro por las ciencias médicas y de la salud (CMS) - , es posible encontrar que 21 de los entrevistados corresponde al bloque de CSHA, mientras que ocho pertenecen a las CMS.

Vale aclarar que en principio no fue considerado incluir investigadores con formación biomédica; sin embargo, permitir la emergencia de categorías de análisis y mantener la apertura de dimensiones en la medida en que iba avanzando la investigación dio lugar a que fueran agregados. Esto generó aportes importantes, puesto que los mismos investigadores 
con formación de base biomédica expresaban la importancia de aprovechar nuevas perspectivas para su trabajo. Por otra parte, reconocían que la integración de perspectivas conceptuales y metodológicas les aportaba en la definición de una posición crítica frente a su propia formación. Podría decirse que estos investigadores con formación de base biomédica hicieron un giro social en sus trayectos de investigación y en la elección de posgrados asociados a las CSHA. Esto queda evidenciado además en la adopción que realizan de marcos conceptuales y metodológicos propios de las CSHA.

Con respecto al enfoque metodológico declarado por los investigadores, resalta la baja presencia del enfoque cuantitativo (solo un investigador), frente a una distribución mayor entre los enfoques cualitativo (16 investigadores) y mixto (11 investigadores). Si bien la elección de enfoques metodológicos y marcos conceptuales varía de acuerdo con el tema o problema abordado, los investigadores siguen una trayectoria sostenida en cierto enfoque, sin que esto quiera decir que no usen otro en una circunstancia particular.

La motivación u orientación que adoptan los investigadores fue clasificada según las respuestas, a partir de tres categorías que toman como referencia la propuesta del Manual de Valencia (OCTS-OEl y RICYT, 2017), y el análisis de Vaccarezza (2009), sobre escisión y compromiso a partir de la «orientación pragmática del investigador» (p. 147). Las tres categorías resultantes fueron motivación/orientación académica, social o comercial, lo que resultó en una mayor presencia de declaraciones enfocadas hacia una integración de interés académico y social ( 22 investigadores), frente a una reducida manifestación de interés exclusivamente académico ( 7 investigadores) y nula presencia de interés comercial.

\section{La salud y la enfermedad como objetos de estudio de investigadores sociales}

Desde la dimensión cognitiva, al indagar por las bases conceptuales que privilegian los investigadores para su trabajo, se evidencia un punto de acuerdo general con respecto al significado de la salud: todos los investigadores la describen como un aspecto asociado a las condiciones de vida y no únicamente al estado biofísico. Las diferencias entre ellos empiezan a marcarse según los abordajes conceptuales y metodológicos específicos y en los referentes que toman como base para sus investigaciones y su ejercicio docente.

Partir del concepto de salud, tanto desde su forma conceptual como práctica, se hace importante, puesto que, como indica Czeresnia (2008), el discurso de la salud está sujeto a trampas. Según la postura de esta 
autora, el concepto de salud no es científico y lo que se ha construido en realidad es el concepto de enfermedad por parte de la ciencia, lo que también representa un proceso de reducción, inevitable para el método científico. Por esta razón, advierte la necesidad de cautela y claridad con respecto a las restricciones existentes; lo que no significa, según la autora, el abandono de conceptos como enfermedad, transmisión o riesgo, sino la oportunidad de analizarlos desde un abordaje más complejo.

Al pensar en una definición de la salud, para los investigadores entrevistados fue ineludible la referencia a la propuesta de la Organización Mundial de la Salud (OMS o WHO, por sus siglas en inglés), considerada por la mayoría como poco realista, puesto que apunta a un tipo de equilibrio y armonía inexistente, si se tienen en cuenta las condiciones de vida. Vale decir que la declaración que aparece en los medios oficiales es la siguiente: «La salud es un estado de completo bienestar físico, mental y social, y no solamente la ausencia de afecciones o enfermedades» (WHO, 1946).

Si bien, como se decía anteriormente, hay una base general y una preocupación central por entender la salud en un sentido más integral, dentro de las posiciones se destacan algunas más moderadas y otras más radicales. Uno de los investigadores afirma: «si no hay salud colectiva, no hay salud» (investigador 2, comunicación personal, 8 de marzo de 2018), en un claro énfasis por la primacía de los aspectos sociales, mientras que desde un punto de vista más moderado otra investigadora representa una posición de complementariedad:

pararte en una vereda desconociendo la otra me parece que no construye [...] empezar a negar que hay cuestiones biológicas o hereditarias involucradas, me parece que no, y algunos autores se paran mucho en eso, y a mí me parece que no contribuye, que no se puede negar tampoco, más bien yo trabajaría en mostrar esto: que la mayor cantidad de factores subyacentes a las enfermedades son de índole social, empezar por allí (investigadora 28, comunicación personal, 16 de enero de 2018).

Los testimonios y la revisión de literatura especializada - en buena parte resultante de las menciones en las entrevistas - proporcionaron un punto de encuentro particular alrededor de la complejidad y lo necesario de un abordaje interdisciplinario. El punto de encuentro entre los estudios de la salud y los estudios de ciencia, tecnología y sociedad logra visualizarse en los aportes de Samaja (2004), un referente mencionado por varios entrevistados e incluso catalogado en el ámbito académico como el «epistemólogo de la salud». Entre las ideas de Samaja está presente la discusión sobre la dicotomía entre contenidos «internos» y «externos» de la ciencia; en este sentido, Samaja muestra lo que podría considerarse como una 
complementariedad entre lo interno/externo, coincidiendo con los constructivistas del campo CTS, quienes han cuestionado la división que se ha planteado al asignársele a la epistemología la responsabilidad por los aspectos internos y a la sociología los externos. Para el caso de la salud, más que epistemología o sociología, un abordaje sociocognitivo muestra la interdependencia de condicionantes biológicos, sociales, políticos, económicos y ambientales. Al decir de Samaja: «entre los contenidos que se evocan como "externos" y los "internos" no hay una mera relación de yuxtaposición, sino de unidad dialéctica o de transformación de unos en otros» (Samaja, 2004, p. 43).

\section{Ciencias sociales y problemas sociales}

Al consultar a los investigadores sobre la configuración de sus agendas de investigación y la decisión sobre qué investigar emergió el contraste entre problemas sociales y problemas de conocimiento (o de investigación). En las ciencias sociales y humanidades parecería haber una mayor cercanía entre estas cuestiones, pero lo que muestran los informantes es que no necesariamente se logra una interacción y realimentación entre los dos tipos de problemas.

La referencia a los problemas sociales además conduce a una interpretación sobre la pertinencia, la utilidad, la crítica hacia los formatos de evaluación y hacia el rol de la universidad, como lo plantea el investigador 5:

Creo que hay una brecha entre la productividad científica o el conocimiento que generamos y la pertinencia de ese conocimiento. Hay una brecha importante, en donde la universidad o los centros de investigación en nuestro país, que principalmente son las universidades, tienen un poco perdido el norte y donde las agendas se circunscriben más a otros intereses que no necesariamente... Incluso son intereses más intradisciplinares y no necesariamente enfocados hacia las necesidades que requieren, en nuestro caso, los pacientes, las poblaciones, los ejes para la transformación social. Y uno lo mira en lo micro, pero necesariamente eso está en una escala global de capitalismo cognitivo, como modelo instaurado, donde la creatividad misma tiene precio, y donde la universidad a la final termina tratando, en el mejor de los casos, de hacer un doble juego: cómo podemos generar que pueda transformar realidades en nuestro contexto, pero también cómo seguimos ese juego a ver si algún día llegamos a ser Harvard, que es una competencia peor (investigador 5, comunicación personal, 8 de febrero de 2018).

Al decir de Kreimer (2011), los problemas sociales o los problemas públicos han sido naturalizados por una buena parte de la tradición de las ciencias sociales, sin una mayor profundización sobre la construcción de 
aquello definido como problema, ya sea como objeto epistémico o como situación objetiva de los actores, y no como una misma operación indiferenciada. Así, por ejemplo, ¿qué hace que la salud sea un tema/problema considerado y de interés para diferentes espacios sociales?, ¿los problemas de salud son problemas sociales?

Esta última pregunta fue realizada a los investigadores participantes en la investigación. Todos asumieron afirmativamente que los problemas de salud eran problemas sociales y se apoyaron en sus bases académicas para darlo por sentado. Sin embargo, que ellos lo consideren así no significa que socialmente o en las agendas pública y política se comprenda de la misma manera. De hecho, el investigador 6 afirmó que las oficinas de gobierno encargadas del sector salud actúan con «lógica de bombero», puesto que solo atienden lo que genera cierta exposición mediática. Más allá de esa circunstancia, opina que la salud no es un tema que importe en gran medida, así esté asociado a la vida diaria y a lo que un investigador social pudiera comprender como parte esencial del desarrollo humano (investigador 6, comunicación personal, 14 de marzo de 2018).

La salud y el entramado complejo que la constituye no necesariamente se refleja o traduce como problema social dentro de las agendas de investigación de los científicos. En el caso de los investigadores de las ciencias sociales y las humanidades resulta más difícil diferenciar esta cuestión; sin embargo, aun así, se encuentra la división propia de los ámbitos social y académico, puesto que cada uno tiene unas dinámicas diferenciadas, que pueden llegar a entrecruzarse, pero no necesariamente a funcionar de forma integrada. Además, en el ámbito académico opera de forma determinante la autonomía de la ciencia y de la universidad, esto bajo la lógica de trabajo sin interferencias externas, de producción intelectual libre de los condicionamientos de ámbitos «externos».

Ante la pregunta que varios investigadores le hicieron en una charla pública a Pierre Bourdieu sobre qué hacer frente a las demandas sociales, el sociólogo francés respondió:

me parece que [ustedes] deberían empezar por afirmar su autonomía, por defender sus intereses específicos, es decir, en el caso de los científicos, las condiciones de cientificidad, etc., y a partir de allí, intervenir en nombre de los principios universales de su existencia y de las conquistas de su trabajo (Bourdieu, 2003, p. 130, citado en Kreimer, 2011, p. 150).

Esta idea es criticada por Kreimer, dada la notoria asimetría de poder que plantea entre los científicos y otros grupos sociales. Mientras Bourdieu pide autonomía para evitar la injerencia de actores externos al campo científico, invita a los científicos a intervenir en otros campos, respaldados 
en el capital simbólico acumulado. Es interesante de considerar en este análisis, dado que las posibilidades de intervención sobre la salud y la enfermedad están condicionadas, en muchas ocasiones, por las maneras en las cuales han sido tematizadas y comprendidas desde el ámbito académico, y generalmente sin la participación de los actores implicados en el problema o desafío social.

\section{Utilidad social del conocimiento científico}

Al preguntarle a los investigadores por el aporte social, derivado de las investigaciones que realizaban, lo que enfatizaban era su aporte disciplinar, la contribución para la generación de nuevo conocimiento, su aprovechamiento en la docencia o en actividades o productos de divulgación científica. Lo que muestra esto no es solo el apego por las figuras o modos tradicionales del ámbito académico, sino también confusiones con respecto al uso del conocimiento. Incluso cuando se les consultaba por actividades que no fueran dirigidas al ámbito académico seguían resaltando los aportes a la docencia derivados de sus investigaciones.

Además, varios investigadores asumían en sus declaraciones la participación en medios de información o la elaboración de formatos divulgativos como parte central de sus actividades de vinculación. En este sentido, Castro-Martínez et al. (2008) explican que la transferencia de conocimiento no incluye las acciones de divulgación o difusión, puesto que estas representan otra función distinta del saber, que corresponde a la transmisión. «Transferencia no es lo mismo que transmisión: dar conferencias al público general o escribir textos divulgativos no es transferir el conocimiento, sino transmitirlo» (p. 622). Aquí radica además una situación de confusión dada por la polisemia que acompaña iniciativas relacionadas con la extensión universitaria.

Sobre transferencia, la investigadora 3 mencionó, a partir de una pregunta por la evaluación a los investigadores, que considera necesaria su inclusión como requisito para la carrera del investigador, sobre todo teniendo en cuenta las situaciones de los países latinoamericanos y las necesidades sociales. Menciona además que cuando trabaja en la producción de artículos científicos trata de asociar sus análisis al mejoramiento de una política pública, a la construcción de indicadores o a la vinculación con organizaciones sociales. Asimismo, cuestiona el sistema de evaluación centrado en la cantidad de artículos de investigación y considera un mayor potencial de contribución en la generación de evidencias para revisar o mejorar alguna situación. «Creo que hay cada vez una articulación mayor, hay equipos interdisciplinarios cada vez mayores. Creo que también desde la academia hay pocas herramientas y poca voluntad a ver 
cómo podemos dialogar con los otros, con los médicos, con los tomadores de decisiones» (investigadora 3, comunicación personal, 23 de febrero de 2018).

Más que transferencia, intercambio. Eso menciona la investigadora 17 al referirse al trabajo conjunto con actores extra-académicos: «el concepto de transferencia o de extensión... son de esos conceptos en los que el que tiene el saber va a transferir o va a extender su saber a la comunidad, jno! Es un intercambio, es un diálogo de saberes diversos» (investigadora 17, comunicación personal, 19 de abril de 2018).

En línea con la interpretación anterior, la investigadora 10, al relatar sus experiencias de trabajo con movimientos y organizaciones sociales, apunta sobre lo que significa el aporte social a partir de las investigaciones: «¿Uno investiga para qué? Yo creo que el uso de lo que uno alcanza a trabajar en investigación responde a la pregunta de para qué quiero yo investigar, porque uno puede quedarse en la universidad 30 años sin hacer nada o dictando clase» (investigadora 10, comunicación personal, 3 de febrero de 2018).

Estas son algunas de las expresiones que dejan ver una perspectiva sobre la utilidad social del conocimiento científico, no solo desde la pregunta, sino desde la reflexión de los investigadores sobre su propio trabajo. No hay que dejar de anotar que las presiones sobre los planes de trabajo en las universidades cada vez se intensifican más. Esto dado que las funciones misionales de docencia, investigación, extensión y gestión se trasladan a la expectativa sobre el desempeño de quienes, en su mayoría, fueron contratados como profesores universitarios, no como investigadores de tiempo completo.

\section{Discusión y conclusiones}

Enfrentar el desafío de «investigar a los investigadores» y de detenerse a revisar en profundidad las dinámicas asociadas a su trabajo - desde sus resultados explícitos, y contemplando el proceso que implica producir y usar conocimientos - da lugar a una mejor comprensión de la configuración de las comunidades disciplinarias, el rol de los investigadores, sus estrategias y agendas de investigación, y su vinculación con otros actores y sectores sociales.

Lo que revela esta investigación, entre otros aspectos, es la persistencia de una tradición académica tradicional abocada a los compromisos ligados a un régimen disciplinar (Shinn, 2008), que muestra poca o nula articulación con actores extraacadémicos. Por otra parte, los investigadores sociales en salud muestran, en la diversidad de sus adscripciones disciplinares, una forma alternativa y en general crítica de abordar la salud 
frente a las tradiciones biomédicas que dominan en los contextos universitarios. A favor, estos investigadores tienen importantes antecedentes en la región latinoamericana; $y$, en contra, una situación de dispersión y poca articulación entre colegas de la investigación social, de la investigación biomédica y de otros ámbitos académicos y sociales.

Los aportes de cada investigador sobre su comprensión de la salud manifiestan las diferentes formas de entenderla, de explicarla. Muestra además las variaciones que tiene su significado en el tiempo y la tensión que aún existe entre lo que Knorr Cetina (2005) llama las «dos ciencias», refiriéndose a la ciencia social y a la ciencia natural. Estas disputas disciplinares se dan en el escenario académico; sin embargo, trascienden a otros espacios, puesto que la formación que reciben los profesionales en las instituciones de educación superior refleja la forma como finalmente se asume la salud. El resultado de esto se ve plasmado en el ejercicio de quienes obtienen un título habilitador, y también de quienes vivencian la salud, utilizan los servicios sanitarios e incluso de quienes participan en la definición de políticas en la materia.

Si bien durante el trabajo se habla de producción y uso de conocimiento científico como dos grandes ejes, se puede notar una imbricación constante, puesto que hay una mutua influencia asociada a la dinámica cognitiva, social y técnica. En el caso de los investigadores sociales dedicados a temas de salud y enfermedad es posible notar - a partir de la indagación sobre sus trayectorias personales, sus bases disciplinares y sus posiciones sobre la salud como asunto o problema social- que la división entre las dinámicas de producción y las de uso son de utilidad para el análisis, pero no necesariamente se encuentran disociadas en la práctica. Esto es más notable aún, considerando que la salud es un tema de gran amplitud y de implicaciones tanto conceptuales como prácticas.

Por otra parte, al aludir a la relación centro-periferia, es posible encontrar las asimetrías en cuanto a producción científica y capacidades necesarias para lograr dicha producción. Además, si se tiene en cuenta que los investigadores considerados en este análisis son de dos países periféricos -no solo en términos económicos, sino en relación con la configuración internacional de las agendas científicas-, se comprende mejor la forma en que se van desarrollando las líneas de trabajo y los aportes resultantes. Particularmente, lo que se destaca en este caso, a partir de los testimonios, es una frecuente referencia a la salud colectiva como una tradición regional, latinoamericana, desmarcada de las tradiciones más institucionalistas o internacionales referidas a la salud pública. Esto muestra indicios de aproximaciones alternativas que están logrando puntos de encuentro entre grupos ubicados en América Latina. 
Sobre la dimensión de utilidad social de la ciencia es posible encontrar que la valoración o desestimación del aporte de los investigadores sociales a la salud dependerá, entre otros aspectos, del sentido de utilidad que le asignen los propios investigadores sociales y otros actores con los que interactúen en el proceso de producción de conocimientos, sean colegas de disciplinas sociales, o de disciplinas naturales, directivos universitarios o actores extraacadémicos. La utilidad no se estima solamente en cuanto a los beneficios recibidos por el campo académico, el sector salud o el estado de salud de las personas, sino a partir de las interacciones y negociaciones que se dan entre dichos sectores o al interior de cada uno. El uso que se le dé al conocimiento producido, e incluso las interacciones dadas en el proceso de producción, darán lugar a determinadas valoraciones sobre su utilidad, ya sea para la carrera académica o para el desarrollo de acciones sociales (Zabala, 2004, p. 164).

En todo caso, los investigadores no son sujetos desinteresados y enfocados solamente en el avance del conocimiento según los criterios del método científico, como lo postulara Merton (1964), sino que son también actores que se desenvuelven en ámbitos diferentes al de la investigación, en los que toman diferentes recursos disponibles (cognitivos, retóricos, políticos, técnicos), según sus intereses y necesidades. De allí que sea necesario para el análisis considerar en un mismo plano la dimensión social y la cognitiva (sociocognitiva), puesto que conviven dentro de un mismo sujeto e intervienen en las decisiones y las formas en que se configura tanto el campo como la carrera académica y las vinculaciones que pueda generar el investigador con otros actores (Zabala, 2004).

En consonancia con lo anterior, la producción científica no deja de ser una forma de producción como las demás, cada vez más sometida, como cualquier otra, a las presiones del mercado o de las instancias políticas. No se trata, como arguye la sociología normativa, de la búsqueda desinteresada del conocimiento científico. A esto hay que agregar que la investigación científica depende estrechamente del apoyo de los poderes públicos y, por lo tanto, está sujeta a cierto control social. En los regímenes democráticos el contribuyente se ve obligado a financiarla, además de responder a los compromisos que cada vez más se adquieren con el sector privado (Salomon, 2008, p. 41).

Finalmente, es importante resaltar que estudiar los procesos de investigación científica y los actores que involucra es una oportunidad para adentrarse en el mundo académico, un espacio lleno tanto de esfuerzos como de tensiones entre colegas, disciplinas y campos académicos, incluso entre ámbitos y sectores sociales. Además, dichos procesos de investigación también están atravesados por intereses académicos y también 
políticos y económicos, que dan lugar a manifestaciones de diverso tipo, tal y como pasa en otros grupos sociales. La academia no está exenta de vicios y perversiones, por más que la imagen de expertos dedicados intente proyectar una visión de sabios intachables e incorruptibles. El interés por profundizar en estas cuestiones se vincula igualmente con dos preguntas que no suelen aparecer explícitas en los protocolos, productos y resultados de investigación: ¿para qué y para quiénes se investiga? Esto conduce a la indagación por la utilidad y el uso social del conocimiento científico. Sin embargo, como queda evidenciado en los aportes conceptuales del campo académico CTS, la utilidad no es un aspecto disociado de la producción, sino que se trata de un proceso dinámico cuyo análisis requiere superar la dicotomía internalista/externalista de la ciencia. En este sentido, es importante tanto la representación que tiene el mismo investigador sobre el uso y la utilidad sobre el conocimiento mientras es producido, como la valoración realizada por actores externos.

En las ciencias sociales y humanidades, este tipo de indagaciones resultan cada vez más necesarias, puesto que sus objetos de estudio suelen estar estrechamente ligados a la sociedad en sus contextos sociales, territoriales, económicos, políticos y culturales. En el caso particular de esta investigación, se complejiza con el objeto salud y su configuración compleja y problemática, dado que es un fenómeno inherente a la vida en su condición psicosocial y biológica. Este trabajo intenta realizar un aporte para ampliar el análisis hacia las ciencias sociales y sus diferentes intereses y desarrollos, puesto que, hasta el momento, el mayor esfuerzo en los estudios de este tipo se ha enfocado hacia las ciencias naturales y sus trayectorias. Esto considerando las limitaciones de este proceso investigativo que podrá ser complementado con nuevos aportes. Resulta importante y necesaria la continuidad de iniciativas habilitadoras de indagación y promoción de instancias reflexivas para pensar y repensar el rol de las comunidades académicas en la sociedad actual, tratando de motivar una apertura inter y transdisciplinar.

\section{Contribución de autoría}

Jorge-Andrés Echeverry-Mejía es el responsable de la elaboración del proyecto de investigación, la recolección de datos, el análisis y la redacción de resultados.

\section{Fuente de financiamiento}

Autofinanciado.

\section{Potenciales conflictos de interés}

Ninguno. 


\section{REFERENCIAS BIBLIOGRÁFICAS}

Bourdieu, P. (2003). El oficio de científico. Ciencia de la ciencia y reflexividad. Anagrama.

Burke, P. (2002). Historia social del conocimiento. De Gutenberg a Diderot. Paidós.

Burke, P. (2012). Historia social del conocimiento. De la Enciclopedia a la Wikipedia: Vol. Il. Paidós.

Castro-Martínez, E., Fernandez-de-Lucio, I., Pérez-Marín, M. y CriadoBoado, F. (2008). La transferencia de conocimientos desde las Humanidades: Posibilidades y características. Arbor, 184(732), 619-636. https://doi. org/10.3989/arbor.2008.i732.211

Congreso de la Nación Argentina. (2001). Ley 25467. Ciencia, tecnología e innovación. Sistema nacional-Régimen legal. http://servicios.infoleg.gob. ar/infoleglnternet/verNorma.do?id=69045

Czeresnia, D. (2008). El concepto de salud y la diferencia entre prevención y promoción. En D. Czeresnia y C. Machado de Freitas (Eds.), Promoción de la salud. Conceptos, reflexiones, tendencias (pp. 47-63). Lugar Editorial.

Echeverry-Mejía, J. A. (2019). Producción y uso social de conocimiento sobre salud. Aproximación al trabajo de investigadores sociales [tesis de maestría inédita]. Universidad Nacional de Quilmes.

Galeano Marín, M. E. (2004). Diseño de proyectos en la investigación cualita-

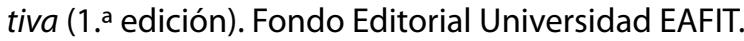

Gibbons, M., Limoges, C., Nowotny, H., Schwartzman, S., Scott, P. y Trow, M. (1994). The new production of knowledge. The dynamics of science and research in contemporary societies. SAGE.

Hernández Sampieri, R., Fernández Collado, C. y Baptista Lucio, M. del P. (2014). Metodología de la investigación (6. edición). McGraw-Hill.

Isoglio, A. y Echeverry-Mejía, J. A. (2018). Ciencia y tecnología a cien años de la Reforma Universitaria: Algunos apuntes sobre la democratización del conocimiento. En M. L. Magallanes Udovicich y A. Zanotti (Eds.), Diálogos en ciencia, tecnología y sociedad. Conocimiento, producción colaborativa, innovación (pp. 101-128). Brujas.

Knorr Cetina, K. (2005). La fabricación del conocimiento. Un ensayo sobre el carácter constructivista y contextual de la ciencia. Universidad Nacional de Quilmes.

Kreimer, P. (2003). Conocimientos científicos y utilidad social. Ciencia, Docencia y Tecnología, 14(26). 
Kreimer, P. (2011). Desarmando ficciones. Problemas sociales-problemas de conocimiento en América Latina. En A. Arellano y P. Kreimer (Eds.), Estudio social de la ciencia y la tecnología desde América Latina (pp. 127-165). Siglo del Hombre. http://bibliotecacts.org/73/

Kreimer, P. y Thomas, H. (2003). Seminario de tesis. Universidad Virtual de Quilmes.

Kreimer, P. y Thomas, H. (2004). Un poco de reflexividad o ¿de dónde venimos? Estudios sociales de la ciencia y la tecnología en América Latina. En P. Kreimer, H. Thomas, P. Rossini y A. Lalouf (Eds.), Producción y uso social de conocimientos. Estudios de sociología de la ciencia y la tecnología en América Latina (pp. 11-89). Universidad Nacional de Quilmes Editorial.

Kreimer, P., Thomas, H., Rossini, P. y Lalouf, A. (2004). Producción y uso social de conocimientos. Estudios de sociología de la ciencia y la tecnología en América Latina. Universidad Nacional de Quilmes Editorial.

Kreimer, P., Vessuri, H., Velho, L. y Arellano, A. (Eds.). (2014). Perspectivas latinoamericanas en el estudio social de la ciencia, la tecnología y la sociedad. Siglo XXI; Foro Consultivo Científico y Tecnológico.

Lamo de Espinosa, E. (2005). ¿Para qué la ciencia social? ¿Para quién escribimos? Nómadas. Critical Journal of Social and Juridical Sciences, 11(1). http://www.redalyc.org/articulo.oa?id=18153294001

Merton, R. (1964). Teoría y estructura sociales. Fondo de Cultura Económica.

MinCyT Argentina. (2017). Indicadores de Ciencia y Tecnología Argentina 2015. Ministerio de Ciencia, Tecnología e Innovación Productiva.

OCTS-OEl. (2018). Informe de Coyuntura (Núm. 1). Observatorio Iberoamericano de la Ciencia, la Tecnología y la Sociedad. http://octs-oei.org/coyuntura/coyuntura01.html

OCTS-OEI y RICYT (Eds.). (2017). Manual iberoamericano de indicadores de vinculación de la universidad con el entorno socioeconómico. Manual de Valencia. http://www.octs-oei.org/manual-vinculacion/

Quiroz Ávila, R. (2018). ¿Las ciencias humanas y sociales no son ciencias? Desde el Sur, 10(1), 7-8. https://doi.org/10.21142/DES-1001-2018-7-8

RICYT y OCTS-OEI. (2018). El estado de la ciencia. Principales indicadores de ciencia y tecnología iberoamericanos / interamericanos (p. 172). Red de Indicadores de Ciencia y Tecnología -Iberoamericana e Interamericana- (RICYT). http://www.ricyt.org/publicaciones

Salomon, J. J. (2008). Los científicos: Entre poder y saber. Universidad Nacional de Quilmes.

Samaja, J. (2004). Epistemología de la salud. Reproducción social, subjetividad y transdisciplina. Lugar Editorial. 
Shinn, T. (2008). Regimes de produção e difusão de ciência: Rumo a uma organização transversaldo conhecimento. Scientiae Studia, 6(1), 11-42. https://doi.org/10.1590/S1678-31662008000100002

Universidad de Antioquia. (1990). Acuerdo Superior 153. Consejo Superior Universitario.

Universidad de Antioquia. (2001). Acuerdo Superior 204. Consejo Superior Universitario.

Universidad de Antioquia. (2011). Acuerdo Superior 386. Consejo Superior Universitario.

Universidad de Antioquia. (2016). Gestión y resultados sociales 2016 (pp. 175-187). Universidad de Antioquia.

Universidad Nacional de Córdoba. (1988). Ordenanza No. 15. Creación de la Secretaría de Ciencia y Tecnología - SECyT. Honorable Consejo Superior.

Vaccarezza, L. S. (2009). Las relaciones de utilidad en la investigación social. Revista Mexicana de Sociología, 71, 133-166.

Vaccarezza, L. S. y Zabala, J. P. (2002). La construcción de la utilidad social de la ciencia. Investigadores en Biotecnología frente al mercado. Universidad Nacional de Quilmes.

Vasen, F. (2018). La «Torre de Marfil» como Apuesta Segura: Políticas Científicas y Evaluación Académica en México. Archivos Analíticos de Políticas Educativas, 26(96), 1-27. http://dx.doi.org/10.14507/epaa.26.3594

Vessuri, H., Guédon, J. C. y Cetto, A. M. (2014). Excellence or quality? Impact of the current competition regime on science and scientific publishing in Latin America and its implications for development. Current Sociology, 62(5), 647-665. https://doi.org/10.1177/0011392113512839

WHO. (1946). Constitution of the World Health Organization. International Health Conference. https://www.who.int/about/frequently-asked-questions

Zabala, J. P. (2004). La utilidad social de los conocimientos científicos como problema sociológico. En P. Kreimer, H. Thomas, P. Rossini y A. Lalouf (Eds.), Producción y uso social de conocimientos. Estudios de sociología de la ciencia y la tecnología en América Latina (pp. 151-172). Universidad Nacional de Quilmes.

Zabala, J. P. (2010). La enfermedad de Chagas en la Argentina: Investigación científica, problemas sociales y políticas sanitarias. Universidad Nacional de Quilmes. 
Zabala, J. P. (2014). Enfermedades, conocimiento y políticas. Nuevas perspectivas en el estudio de los problemas sociales. En P. Kreimer, H. Vessuri, L. Velho y A. Arellano (Eds.), Perspectivas latinoamericanas en el estudio social de la ciencia, la tecnología y la sociedad (pp. 396-398). Siglo XXI y Foro Consultivo Científico y Tecnológico.

Recepción: 2/3/2021

Aceptación: 18/6/2021 